\title{
Nonlinearity Management of Fiber Oscillator with Multiple Gain Segments
}

\author{
Tesfay G. Teamir ${ }^{1, *}$ and F. Ömer Ilday ${ }^{1,2}$ \\ 1. Department of Physics, Bilkent University, Ankara, 06800, Turkey \\ 2. Department of Electrical and Electronics Engineering, Bilkent University, Ankara, 06800, Turkey \\ *teamir@fen.bilkent.edu.tr
}

\begin{abstract}
Oscillator with two gain segments is used to manage nonlinearity level and distribution. Results suggest that pulse evolution subject to an effective negative nonlinearity arising from complex interaction of gain filtering, dispersion, SFM and chirp.

OCIS codes: (060.2270) Fiber characterization; (190.5940) Self-action effects; (320.5540) Pulse shaping.
\end{abstract}

\section{Introduction}

Shortly after nonlinearity was understood as a route for energy scaling in soliton era, discovery of similariton and dissipative soliton evolution regimes upgraded energy scaling of single mode fiber (SMF) based oscillators to subhundred and recently to hundreds of nanojoules [1,2]. Nonlinear phase accumulation engender pulse energy limit and achievable shorter pulse duration hence it is treated as abrasive physical process. Avoiding nonlinearity by using Large mode area photonic crystal gain fibers (LMAPCF), chirped and divided pulse amplification, and Nonlinear pulse shaping of arbitrary pulses in to parabolic profile pushed the scaling farther more to micro joule regime with megawatt peak power [3, 4].

On the other hand pulse generation and dynamics in different mode locking regimes are determined by how nonlinearity is interacting with the remaining physical process [5]. Optimum levels of nonlinearity was also observed to balance or compensate other physical process such as GVD, third order dispersion (TOD) and gain filtering in fiber lasers $[6,7]$. Generally one can say the nonlinear property of fibers make it a seminal platform for fundamental research as well as interesting applications that could not be provided with other optical systems. Hence, understanding the level and distribution of nonlinearity while designing optical fiber systems always worth a prominent value [8].

In this paper the effects of nonlinearity level and distribution of a fiber oscillator with double gain pumping is investigated. The cavity is characterized on three different pulsing regimes. A similar simulation is done which shows possible pulse evolution that can be supported by oscillator with the same parameters as our experiment. The effect of chirp on the interaction of GVD with SPM is also examined in addition to possible generation of effective negative nonlineaity.

\section{Experimental and simulation results}

The oscillator shown on the first part of figure 1 contains a total of $\sim 520 \mathrm{~cm}$ with repetition rate of $26.2 \mathrm{MHz}$. It has a two segments of $\mathrm{Yb}$-doped gain fiber pumped at different positions for management of nonlinearity level and distribution in the cavity.

As can be seen in the second (II) part of figure 1 below the spectral width of a pulse is compared from three out output coupling ports. It is found that the spectral width after gain 2 is always smaller than the spectral width after gain 1 in all pulsing regimes characterized by total dispersion indicated on the figure even when its width is smaller than the gain bandwidth. The total pump power is kept almost constant while pump powers on each segment varies (II (d)). With This approach the nonlinearity, which can be described by nonlinear phase shift, changes in its level and distribution over fiber components. Duration of externally compressed pulse output from PBS varies with variation of the nonlinearity. It can be recompressed back with smaller or larger external dispersion delay line (DDL) in to almost the same transform limited pulse width. Spectral width of the pulse was increasing with pumping at gain 2 but it is almost not responding to the pumping on gain 1. Due to significant length of passive fiber between the two gain fibers, nonlinearity is larger when pumping on gain 1 is larger. Therefore, increasing of spectral width with pump power 2 cannot be fully explained with interaction of gain filtering and SPM only.

To support our experimental results, we have done simulation based on solution of generalized nonlinear Schrödinger equation (details are explained on reference [10]). The results are indicated on part (III) of figure 1. As 
(I)

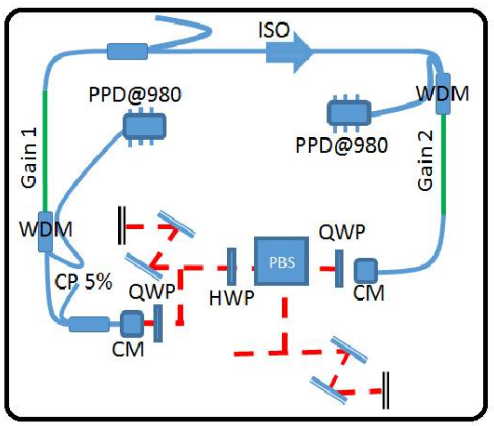

(II)
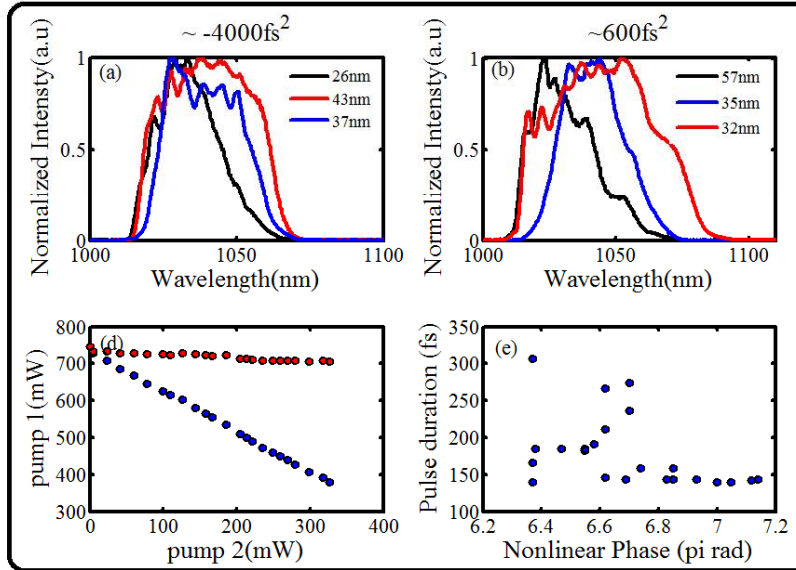

Wavelength(nm)

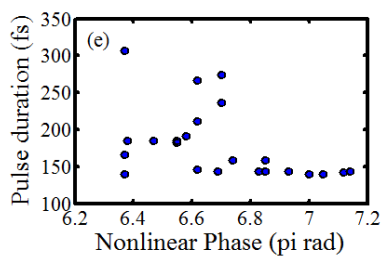

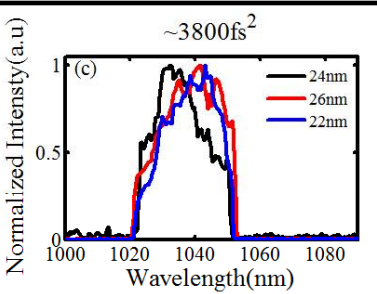

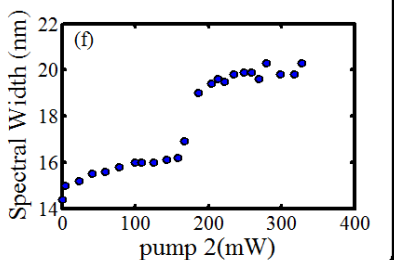

(III)
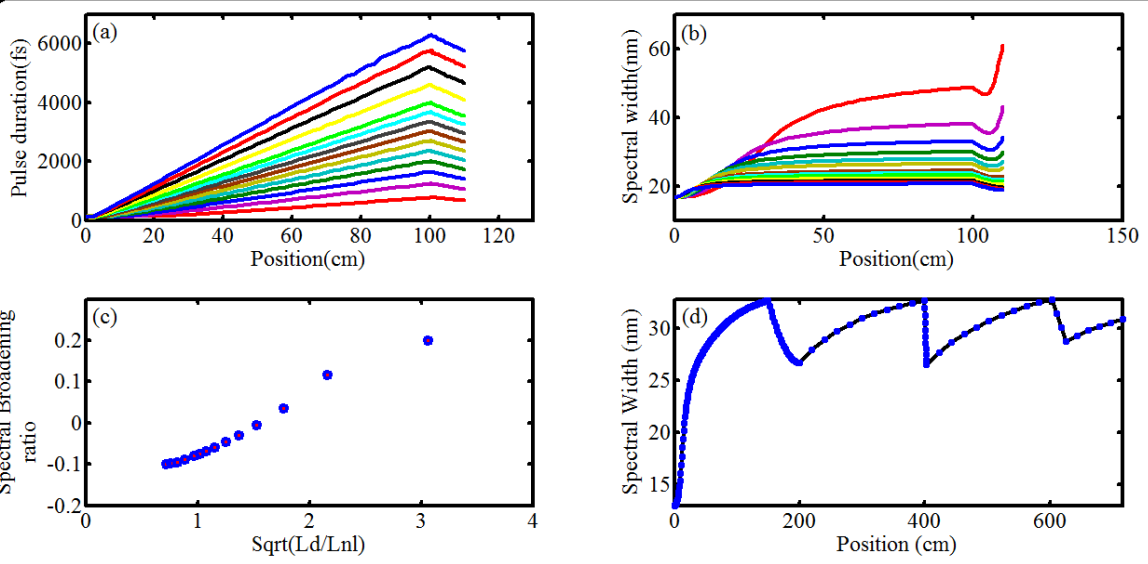

Stretched Pulse

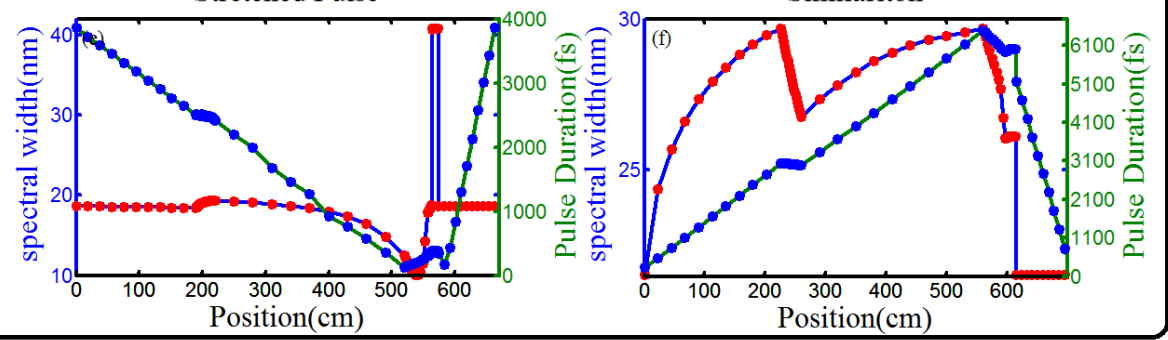

Fig 1. I. Experimental set up, II. Experimental results (a-c) spectral width of the pulse at different outputs. (d-f) a constant total power crating different nonlinearity level and distribution with its effect on duration and spectral width of the pulse. III. Simulation results. (a-c the chirp increases from $10-110 \mathrm{fs}^{\wedge} 2 / \mathrm{mm}$ in the first segment) effects of a chirped pulse initiating the interaction of SFM and positive GVD. (d-f) initiation of effective nonlinearity on gain fibers inside oscillator and cascade of passive and active fibers. 
shown theoretically in reference [9], III (a-c) suggested that large chirp on a pulse can initiate compensation of SFM with positive GVD. III (e, f) shows stretched and similariton pulse evolution can be supported by a cavity with the same parameters as our oscillator. By varying nonlinearity on the two gain segment we able to switch in between the two pulse evolutions. Negligible spectral expansion in $1^{\text {st }}$ gain of stretched pulse and spectral compression in the two gain fibers of similariton pulse evolution similar to our experimental result can be explained by the generation of effective negative nonlinearity. This idea can even be more extended with cascade of passive and active fibers (III (d)), in which the spectral behavior in each segment is controlled by managing nonlinearity distribution on it.

\section{Conclusion}

In conclusion, we show through simulations and experiments that pulse evolution can be controlled to a certain extend through the distribution of nonlinear phase accumulation inside the oscillator. We observe that pulses with large chirp can undergo spectral compression in gain fiber while it has a positive increasing chirp and collective action of sequentially arranged active and passive fibers can initiate spectral compression as shown in Fig 1(III(e,f) for oscillator) and Fig 1(III(d) for cascade of gain and passive fiber) by managing distribution of nonlinear phase accumulated in each segment. This is a result of the complex interaction between large pulse chirp, gain filtering, dispersion and SPM. Thus, one can have an effective negative nonlinearity by artificial arrangement of gain and passive fibers, akin to metamaterials controlling the linear refractive index. The dynamics explored here, while preliminary, have exciting implications for energy scaling and limitations to pulse durations of mode-locked oscillators.

\section{Acknowledgment}

This work is supported by The Scientific and Technological Research Council of Turkey under grant TÜBITAK $1001-113 F 319$.

\section{References}

[1] A. Chong, J. Buckley, W. Renninger, and F. Wise, "All- normal-dispersion femtosecond fiber laser" Opt. Express 14, 21, (2006).

[2] F.O. Ilday and F. W. Wise,"Nonlinearity management: a route to high-energy soliton fiber lasers", J. Opt. Soc. Am. B, 19, (2002).

[3] F. Rser, J. Rothhard, B. Ortac, A. Liem, O. Schmidt, T. Schreiber, and J. Limpert, "90 W average power 100 J energy femtosecond fiber Chirped-pulse amplification system", Opt. Express, Vol. 30, No. 20 (2005).

[4] S. Boscolo, A.I. Latkin, and S.K. Turitsyn, "Passive nonlinear pulse shaping in normally dispersive fiber systems", IEEE JOURNAL OF Qua. Elec., 44, (2008).

[5] B. Oktem1, C. 1gdr and F. Ilday, "Solitonsimilariton fibre laser", Nature Photonics 4, 307 - 311 (2010).

[6] S.Zhou, L. Kuznetsova, A. Chong, and F. Wise,"Compensation of nonlinear phase shifts with third-order dispersion in short-pulse fiber Amplifiers", Opt. Express, Vol. 13, No. 13, (2005).

[7] D.N. Schimpf, E. Seise, J. Limpert, and A. Tunnermann,"Self-phase modulation compensated by positive dispersion in chirped-pulse systems", Opts. Expr., Vol. 17, No. 7 (2009).

[8] F. O. Ilday, and F. W. Wise, "Nonlinearity management: a route to high-energy soliton fiber lasers," J. Opt. Soc. Am. B 19, 470 (2002).

[9] Schimpf, E. Seise, J. Limpert, and A. Tnnermann, "Self-phase modulation compensated by positive dispersion in chirped-pulse Systems," Opt. Exp, 17, 4997-5007 (2009).

[10] B. Oktem, C. U“ Igudur and F. O. Ilday, “Soliton-similariton fibre laser,” Nature Photon., 4, 307 (2010). 